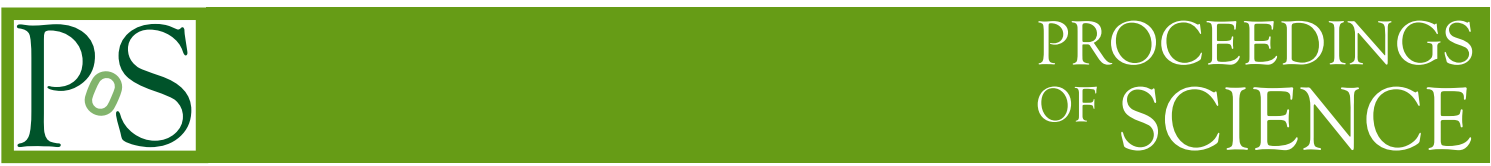

\title{
CMS Run 2 High Level Trigger Performance
}

\author{
Hale Sert* on behalf of the CMS Collaboration \\ RWTH Aachen University, Experimental Physics Institute 3B \\ E-mail: hale.sertecern.ch
}

The Compact Muon Solenoid (CMS) experiment selects events with a two-level trigger system, the Level-1 (L1) trigger and the High Level trigger (HLT). The HLT is a farm of approximately $30 \mathrm{~K}$ CPU cores that reduces the rate from $100 \mathrm{kHz}$ to about $1 \mathrm{kHz}$. The HLT has access to the full detector readout and runs a streamlined version of the offline event reconstruction. In LHC Run 2 the peak instantaneous luminosity reached values above $2 \times 10^{34} \mathrm{~cm}^{-2} \mathrm{~s}^{-1}$, posing a challenge to the online event selection. An overview of the object reconstruction and trigger selections used in the 2016-2018 data-taking period will be presented. The performance of the main trigger paths and the lessons learned will be summarised, also in view of the coming LHC Run 3.

European Physical Society Conference on High Energy Physics - EPS-HEP2019 -

10-17 July, 2019

Ghent, Belgium

${ }^{*}$ Speaker. 


\section{High Level Trigger in CMS Experiment}

High Level Triggers (HLT) in CMS [1] are designed as a menu made of more than 400 different paths targeting a broad range of physics signatures and purposes. Each HLT path consists of a sequence of reconstruction and filtering modules arranged in increasing complexity. The faster algorithms run first and the time consuming algorithms, which are mostly the ones for a reconstruction similar to offline, are run at the end of the path. If a filter fails during reconstruction, the remaining part of the path is skipped in order to keep the CPU time under control.

Processing time of an HLT path is limited due to computing resources, therefore there are some simplifications applied in the online reconstruction. One of them is the intermediate selection steps before running CPU time consuming reconstruction parts. These steps use the information taken only from calorimeters, pixel tracks or muon detectors to filter events. In addition, tracking follows a simplified version of the offline tracking. Furthermore, reconstruction of many trigger objects are performed regionally in a specific region of the detector instead of global volume.

\section{Tracking at HLT}

The online reconstruction of tracking [2] uses a simplified version of offline tracking [3, 4] with reduced number of iterations and regional tracking in some of the iterations. The track reconstruction algorithm was updated at the end of 2016 data-taking due to phase 1 upgrade of the pixel detector, where the number of layers were increased by one layer in both barrel and endcap [5]. The algorithm was updated to start the seeding with quadruple pixel hits instead of triplets as it was done in 2016. Tracking with this update provided much lower fake rates and higher efficiencies especially in the endcap region [6]. During 2017 data-taking, the pixel detector experienced some broken modules that cause reduction in the online tracking efficiencies. A recovery sequence seeding with pixel doublets is added to the existing three tracking iterations for specific $\eta-\phi$ regions. The recovery was updated to a dynamic one in 2018, where it checks the problematic regions automatically. Figure 1 shows the performance of HLT tracking with the recovery sequence. The doublet recovery sequence recovers most of the efficiency loss and brings the efficiency very close to the perfect detector case.
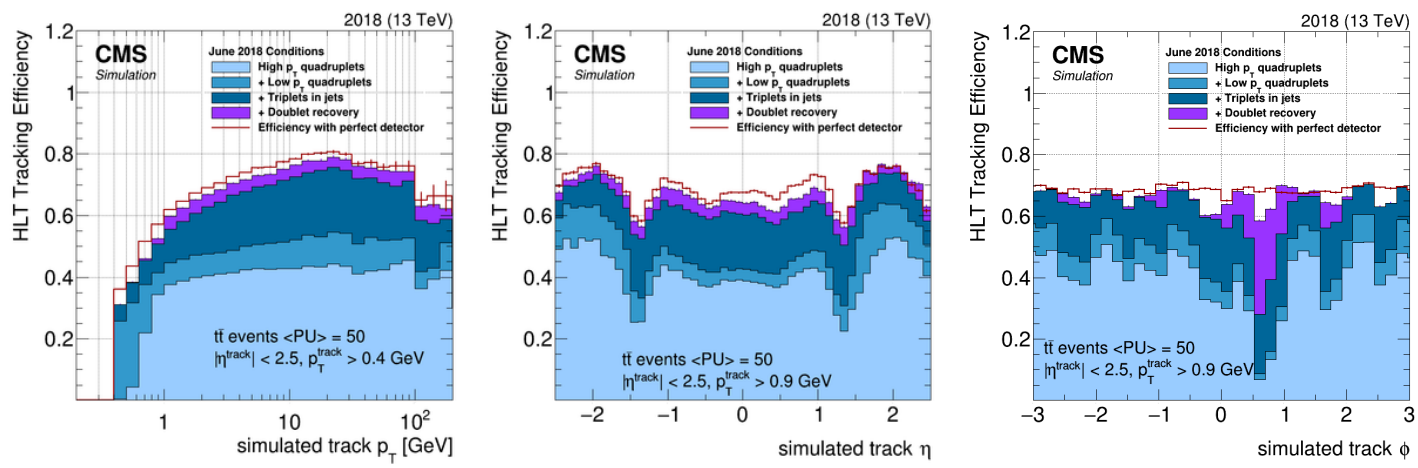

Figure 1: HLT tracking efficiencies as a function of simulated track $p_{T}$ (left), $\eta$ (middle) and $\phi$ (right) for the original three tracking iterations and the doublet recovery compared with the perfect detector case, where there is no pixel modules considered broken [13]. 


\section{Object Reconstruction HLT}

Electrons: Electron reconstruction at HLT starts with building up superclusters (SC) from the energy depositions in the electromagnetic calorimeter. The hits in the pixel detector are matched with the supercluster. The matched SC are used as seed for track reconstruction that uses the Gaussian Sum Filter (GSF) algorithm [4]. Due to the phase 1 pixel upgrade, the pixel matching algorithm was changed from doublets to triplets and retuned in 2017. In addition, various retuning of isolation/identification during Run 2 was performed to cope with the evolving data-taking conditions. Figure 2 presents the performance of electron triggers during Run 2. The left plot compares trigger efficiencies of the electron with $p_{T}=23 \mathrm{GeV}$ of a double electron trigger with $p_{T_{1}}=23$ $\mathrm{GeV}$ and $p_{T_{2}}=12 \mathrm{GeV}$ before and after the pixel phase 1 upgrade. It is seen that the trigger efficiency is reduced with the new pixel detector, however it reduces the rate as well by $70 \%$ [7]. The right plot in Figure 2 presents the performance of single electron trigger with $p_{T}=32 \mathrm{GeV}$ for the last part of data taken in 2016 and 2017, where the working points are modified to gain from the reduction of the rate in 2017 with the new pixel detector. The plot shows the gain in the endcap region from the modification of the working point, which makes the dependency on $\eta$ flatter.
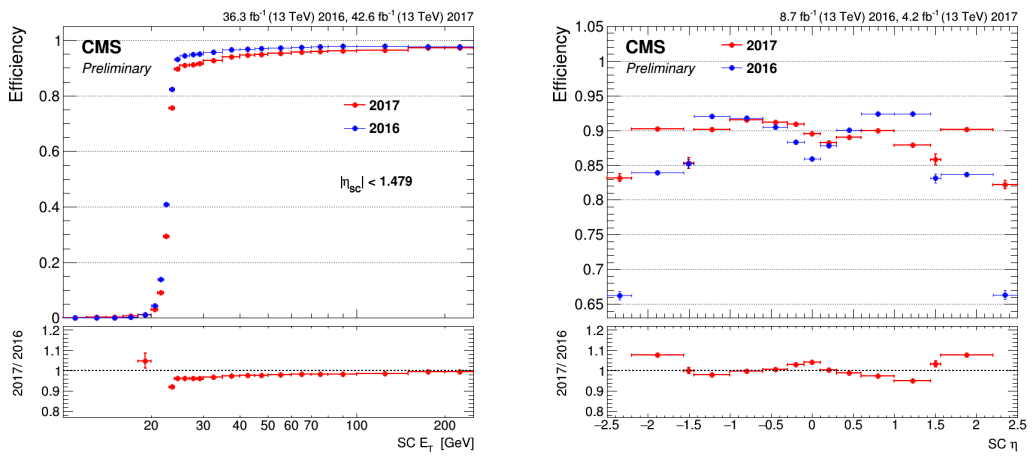

Figure 2: Left: Trigger efficiency of the electron with $p_{T}=23 \mathrm{GeV}$ of double electron trigger with $p_{T_{1}}=23 \mathrm{GeV}$ and $p_{T_{2}}=12 \mathrm{GeV}$. Right: Trigger efficiency for a single electron trigger with $p_{T}=32 \mathrm{GeV}$ with a modification in the working point in 2017 [13].

Muons: Muon reconstruction is performed in two steps at HLT. In the first step, the so-called L2 muons are reconstructed using the information only from muon detectors. In the next step, the L3 reconstruction is performed by exploiting the full detector information. In 2016, there were two different L3 reconstruction algorithms, cascade and tracker muon, used to reconstruct two different muon trigger paths [8]. The "OR" of these two trigger paths gave the best performance in 2016, where one can see the performance in Ref. [6]. The two algorithms were combined in 2017, which relies on the iterative tracking with quadruplet and triplet pixel hits [9]. In addition to this significant change, there were further updates in 2018, such as adding one more iteration with doublet hits and adding a simple identification to keep the high purity with lower rate. Figure 3 shows the improvements in the efficiency as a function of $p_{T}$ and $\eta$ of offline muons and $N_{\text {vertices. }}$. The figure represents that the updates improve the efficiency in the whole $p_{T}$ region, especially at low $p_{T}$, and in the overlap region of two different gaseous detectors, as well as provide a higher efficiency at high pileup. 

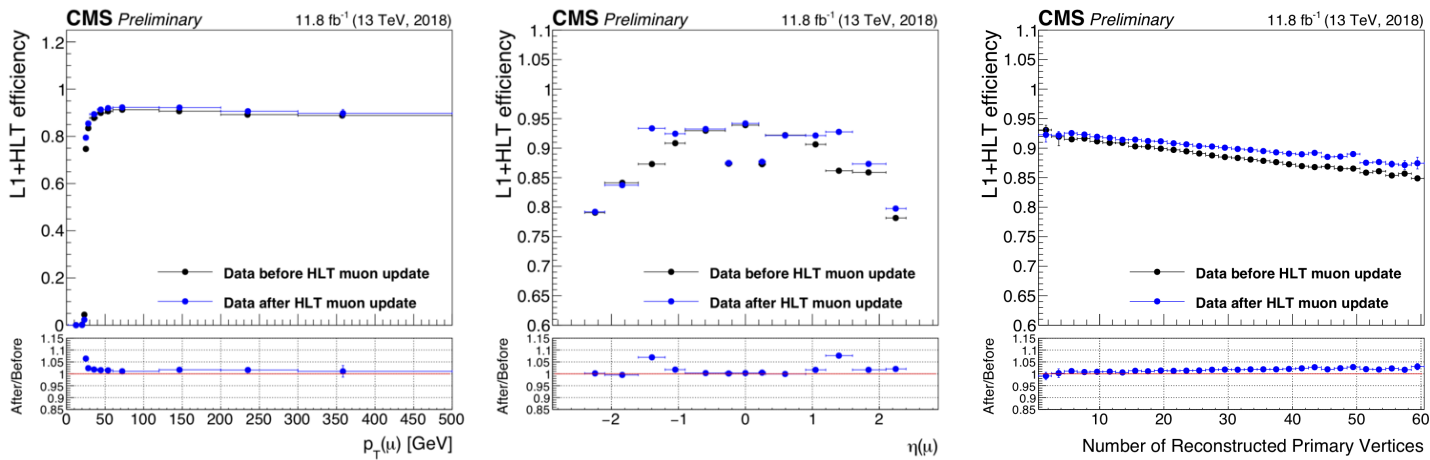

Figure 3: Trigger efficiencies of isolated single muon with $p_{T}=24 \mathrm{GeV}$ as a function of $p_{T}$ (left) and $\eta$ (middle) of offline muons as well as $N_{\mathrm{vtx}}$ (right). The plots compares the performances before and after the updates performed in 2018 data-taking [13].

Tau Leptons: Tau leptons decaying into hadrons, $\tau_{h}$, are reconstructed at HLT using the particle flow (PF) algorithm [4] globally or regionally depending on the type of tau lepton triggers. $e \tau_{h}$ and $\mu \tau_{h}$ triggers are reconstructed globally, while di- $\tau_{h}$ triggers use regional PF reconstruction around the L1 $\tau_{h}$ candidate. In the case of di- $\tau_{h}$ triggers, two more filters by using the jets reconstructed from the calorimeter information only and track based isolation are applied to reduce the CPU time. In the final step, the so-called L3 reconstruction, tau leptons were reconstructed by using the cone-based algorithm until 2018, where there is no separation between decay modes. The reconstruction was updated to hadron-plus-strip (HPS) algorithm [10] in 2018 that makes the separate decay mode reconstruction possible in online and which aligns it with the offline reconstruction. The HPS-based algorithm provides better $p_{T}$ resolution as seen from Figure 4 (left), and reduces the rate of tau lepton trigger by $10 \%$ per tau-leg [11]. The middle and right plots of Figure 4 represent the performance of $\mu \tau_{h}$ trigger in Run 2. The HLT and L1 $p_{T}$ thresholds and isolation of tau leptons were changed during Run 2 data-taking period, and this was reflected in different shapes, especially between 2016 and others. The overall efficiency in 2017 is seen lower than the other years, which is mainly coming from the pixel issues experienced.
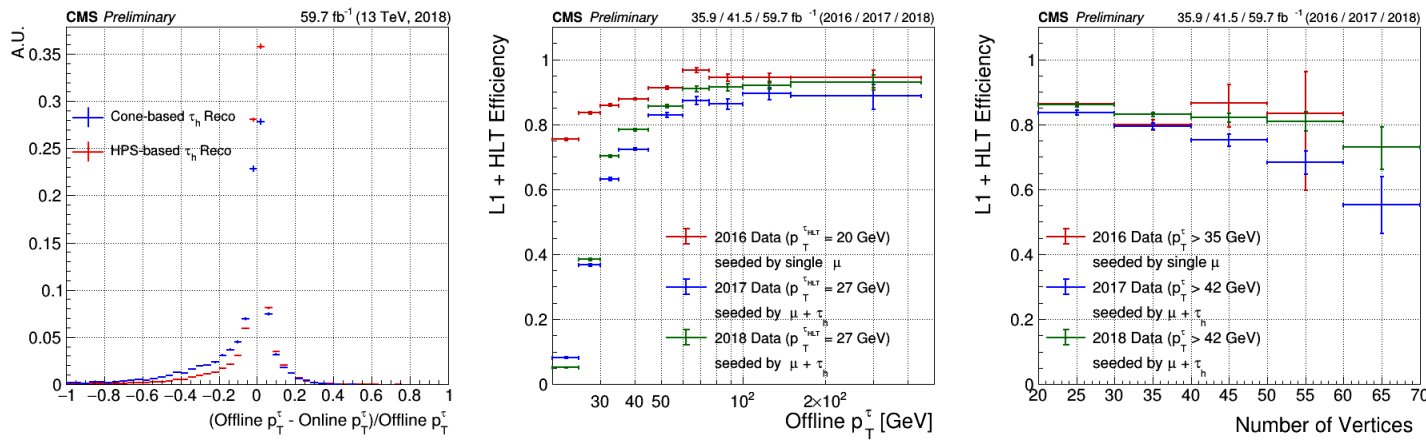

Figure 4: Left: $p_{T}$ resolution of two different reconstruction algorithms of tau leptons used in 2018 data-taking. Middle and Right: Trigger efficiencies of 2016, 2017 and 2018 data-taking as a function of $p_{T}$ of offline tau leptons and of number of vertices [13]. 
JetMET: JetMET online reconstruction starts with the reconstruction of the jets and METs by using the calorimeter information as a first step to filter the events out in order to reduce the CPU time. Then PF reconstruction is performed using the full detector information. The PF reconstruction used was aligned with the offline reconstruction starting from 2016. During Run 2 data-taking, there were some updates in the reconstruction in order to cope with noise. In addition, the calibration methods used in jet energy correction and PF hadron calibration where the calibration is applied in order to take into account the different responses of ECAL and HCAL were updated and aligned with the offline. The jetMET HLTs provided consistent results with high performance during Run 2 [13].

B Tagging: $b$-jets are identified at HLT by using a b-jet tagging algorithm which is Combined Secondary Vertex (CSVv2) [12] up to 2018 and DeepCSV [12] in 2018. The CSVv2 algorithm showed 5\% lower efficiency in online identification compared to the offline one [6,13]. The b-jet tagging is performed in two different ways: one performs the tracking in the PF sequence, while the other performs the tracking regionally around the leading calorimetric jets. The regional tracking reconstruction around calojets reduces the computing time by approximately $75 \%$ [13].

\section{Summary \& Outlook}

Many developments were performed during Run 2 to improve the reconstruction of HLT objects and also to mitigate the experienced issues during data-taking. The HLTs at CMS performed well and maintained high performance in Run 2. In Run 3, there is the potential possibility to use GPUs at HLT which would improve the performance with more precise tracking.

\section{References}

[1] CMS Collaboration, "The CMS trigger system", JINST 12 (2017) P01020.

[2] M. Tosi, "Tracking at High Level Trigger in CMS", Nuclear and Particle Physics Proceedings Volumes 273-275 (2016) Pages 2494-2496.

[3] CMS Collaboration, "Description and performance of track and primary-vertex reconstruction with the CMS tracker", 2014 JINST9 P10009.

[4] Sirunyan, A. M. and others, "Particle-flow reconstruction and global event description with the CMS detector", CMS-JINST-12-2017-10-P10003.

[5] CMS Collaboration, "CMS Technical Design Report for the Pixel Detector Upgrade", CMS-TDR-11, CERN-LHCC-2012-016 (2012).

[6] S. Donato, "CMS Trigger Performance", EPJ Web Conf. 182 (2018) 02037.

[7] CMS Collaboration, "Electron trigger performance in CMS with the full 2017 data sample", CMS DP-2018/030.

[8] P. Verwilligen, "Muons in the CMS High Level Trigger System", Nuclear and Particle Physics Proceedings Volumes 273-275 (2016) Pages 2509-2511.

[9] K. Lee, "Muon Performance with CMS detector in Run2 of LHC", PoS ICHEP2018 (2019) 690.

[10] CMS Collaboration, "Performance of $\tau$-lepton reconstruction and identification in CMS", JINST 7 (2012) P01001.

[11] CMS Collaboration, "2018 Tau Trigger Reconstruction Comparison", CMS DP-2018/035.

[12] CMS Collaboration, "Identification of heavy-flavour jets with the CMS detector in pp collisions at 13 TeV", JINST 13 (2018) P05011.

[13] CMS Collaboration, "High Level Trigger Run 2 Results", https://twiki.cern.ch/twiki/bin/view/CMSPublic/HighLevelTriggerRunIIResults. 